\title{
Static Deformations of a Linear Elastic Porous Body Filled with an Inviscid Fluid
}

\author{
F. DELL'ISOLA ${ }^{1}$, G. SCIARRA ${ }^{2}$ and R.C. BATRA ${ }^{3}$ \\ ${ }^{1}$ Dip. Ingegneria Strutturale e Geotecnica, Universita degli Studi di Roma "La Sapienza", \\ via Eudossiana 18,00184 Roma, Italy.E-mail: Francesco.Dellisola@uniroma1.it \\ ${ }^{2}$ Dip. Ingegneria Chimica, dei Materiali, delle Materie Prime e Metallurgia, Università degli Studi \\ di Roma "La Sapienza”, via Eudossiana 18,00184 Roma, Italy.E-mail: giulio.sciarra@ uniroma1.it \\ ${ }^{3}$ Department of Engineering Science and Mechanics, M/C 0219, Virginia Polytechnic Institute and \\ State University, Blacksburg, VA 24061,U.S.A. E-mail: rbatra@vt.edu
}

\begin{abstract}
We study infinitesimal deformations of a porous linear elastic body saturated with an inviscid fluid and subjected to conservative surface tractions. The gradient of the mass density of the solid phase is also taken as an independent kinematic variable and the corresponding higher-order stresses are considered. Balance laws and constitutive relations for finite deformations are reduced to those for infinitesimal deformations, and expressions for partial surface tractions acting on the solid and the fluid phases are derived. A boundary-value problem for a long hollow porous solid cylinder filled with an ideal fluid is solved, and the stability of the stressed reference configuration with respect to variations in the values of the coefficient coupling deformations of the two phases is investigated. An example of the problem studied is a cylindrical cavity leached out in salt formations for storing hydrocarbons.
\end{abstract}

Key words: solid-fluid mixture, conservative tractions, principle of virtual power, partial tractions, fluid-filled cylindrical cavity, stability analysis.

R.C. Batra dedicates this work with deep respect and admiration to Professor C.A. Truesdell, a superb teacher and an excellent friend.

\section{Introduction}

Simple models of a mechanical system comprised of a deformable porous solid matrix filled with a compressible fluid have been developed by Fillunger [22], Biot [11], Truesdell [40] and Müller [30]. In these works a spatial point is simultaneously occupied by all constituents. This is readily comprehensible for gaseous mixtures [30] and fluid solutions [41].

Observations on fluid saturated solids have shown higher values of fluid percolation through pores of the solid matrix than that predicted by the aforestated 
models (see, e.g., [13] or [33]). The increase of percolation is possibly not only due to the higher externally applied pressure but also due to the opening of pores in the vicinity of the boundary (see [17]). This provides a justification for endowing the theory of mixtures with the volume fraction concept (see, e.g., [18]); this additional scalar parameter can describe the nonstandard dilatation effects (see, e.g., [38]) as follows. Once the mixture is modelled from a microscopic point of view and the reference configuration of the solid matrix is required to be periodic, then those dilatations of pores which do not involve global deformations of cells are captured by the volume fraction.

In this paper we consider a binary mixture involving a second gradient solid and a perfect fluid; we refer the reader to [23, 24, 28] for the relationship between micro-structural and second gradient theories. Our approach is close to that of the volume fraction concept as the latter reduces to the former once a suitable constraint among the enlarged set of state parameters is assumed [34]. For example, for an incompressible solid constituent, it is easy to see [25] how a mixture model endowed with the volume fraction concept transforms into a binary mixture whose solid constituent has second gradient constitutive relations. After formulating a general problem, we study deformations of a porous hollow linear elastic cylinder filled with a perfect fluid. In particular, by assuming that the internal energy density can be split into a part involving first gradient of the displacements and a part involving second order gradients of displacements, we perform parametric analysis of the density profiles of the solid matrix with respect to a suitable energetic coupling coefficient between the solid and the fluid. We limit our analysis to the case when the external tractions applied on both constituents are conservative and can be derived from a potential.

We also discuss stability of the prestressed reference configuration of the hollow cylinder with respect to perturbations of the aforementioned energy coupling coefficient; an energetic criterion is proposed for this analysis. The distance in the space of mixture configurations is described in terms of the total energy which equals the sum of the mixture deformation energy and the potential of surface tractions.

Batra et al. [5-9] have analyzed numerically finite transient thermomechanical deformations of a homogeneous body with the Cauchy stress and higher-order stresses depending upon gradients of deformation.

\section{Formulation of the Problem}

Material particles of the fluid and the solid are identified respectively by their position vectors $\mathbf{X}^{(\mathrm{f})}$ and $\mathbf{X}^{(\mathrm{s})}$ in fixed reference configurations $\Omega_{0}^{\mathrm{f}}$ and $\Omega_{0}^{\mathrm{s}}$. We presume that, at any time $t$, particles of both constituents occupy the same position $\mathbf{x}$ in the present configuration $\Omega$. The velocity $\mathbf{v}^{(\alpha)}(\alpha=\mathrm{f}, \mathrm{s})$ of the material particle $\mathbf{X}^{(\alpha)}$ is defined by

$$
\mathbf{v}^{\alpha}=\frac{\mathrm{d}^{(\alpha)} \mathbf{u}^{(\alpha)}\left(X^{(\alpha)}, t\right)}{\mathrm{d} t}
$$


where $\mathrm{d}^{(\alpha)} / \mathrm{d} t$ denotes the material time derivative following the motion of $\mathbf{X}^{\alpha}$ and $\mathbf{u}^{(\alpha)}$ is the displacement of the $\alpha$ th constituent from its reference configuration. Let $\rho^{(\mathrm{f})}$ and $\rho^{(\mathrm{s})}$ denote, respectively, the apparent mass densities of the fluid and the solid; then the mass density $\rho$ of the mixture equals $\rho^{(\mathrm{f})}+\rho^{(\mathrm{s})}$. The mean or the barycentric velocity $\mathbf{v}$ of the mixture is defined by

$$
\rho \mathbf{v}=\rho^{(\mathrm{f})} \mathbf{v}^{(\mathrm{f})}+\rho^{(\mathrm{s})} \mathbf{v}^{(\mathrm{s})} .
$$

Details of the theory of mixtures are given in [13, 19, 27, 29-31, 33, 40].

\subsection{BALANCE LAWS}

We presume that there is no interconversion of mass between the solid and the fluid. In the spatial description of motion, the balance of mass for each constituent is given by

$$
\frac{\mathrm{d}^{(\alpha)} \rho^{(\alpha)}}{\mathrm{d} t}+\rho^{(\alpha)} \operatorname{div} \mathbf{v}^{(\alpha)}=0
$$

where $\operatorname{div} \mathbf{v}=\operatorname{tr}(\operatorname{grad} \mathbf{v})$, and grad denotes derivatives with respect to coordinates in the present configuration. We use the principle of virtual power to derive the balance of linear momentum and the boundary conditions for each constituent. That is, we postulate that

$$
\begin{aligned}
& \int_{\Omega}\left(\mathbf{m}^{(\mathrm{s})} \cdot \overline{\mathbf{v}}^{(\mathrm{s})}+\mathbf{m}^{(\mathrm{f})} \cdot \overline{\mathbf{v}}^{(\mathrm{f})}+\mathbf{T}^{(\mathrm{s})} \cdot \nabla \overline{\mathbf{v}}^{(\mathrm{s})}-p^{(\mathrm{f})} \operatorname{div} \overline{\mathbf{v}}^{(\mathrm{f})}+\Pi^{(\mathrm{s})} \cdot \nabla \nabla \overline{\mathbf{v}}^{(\mathrm{s})}\right) \mathrm{d} V \\
& =\int_{\Omega}\left(\mathbf{b}^{(\mathrm{s})} \cdot \overline{\mathbf{v}}^{(\mathrm{s})}+\mathbf{b}^{(\mathrm{f})} \cdot \overline{\mathbf{v}}^{(\mathrm{f})}\right) \mathrm{d} V \\
& \quad+\int_{\partial \Omega}\left(\mathbf{t}^{(\mathrm{s})} \cdot \overline{\mathbf{v}}^{(\mathrm{s})}+\mathbf{t}^{(\mathrm{f})} \cdot \overline{\mathbf{v}}^{(\mathrm{f})}+\boldsymbol{\tau}^{(\mathrm{s})} \cdot \frac{\partial \overline{\mathbf{v}}^{(\mathrm{s})}}{\partial n}\right) \mathrm{d} A
\end{aligned}
$$

Here $\mathbf{m}^{(\alpha)}$ is the bulk solid-fluid interaction force, $\mathbf{T}^{(\mathrm{s})}$ the partial Cauchy stress in the solid, $p^{(\mathrm{f})}$ the hydrostatic pressure in the fluid (we assume that the fluid is ideal; therefore partial Cauchy stress in it is spherical), $\Pi^{(\mathrm{s})}$ the second-order stress in the solid, $\nabla$ the gradient operator with respect to coordinates in the present configuration, $\mathbf{b}^{(\alpha)}$ the density of partial body forces, $\mathbf{t}^{(\alpha)}$ the partial surface tractions, $\boldsymbol{\tau}^{(\mathrm{s})}$ the traction corresponding to the second-order stress tensor in the solid, $\overline{\mathbf{v}}^{(\mathrm{s})}$ the virtual velocity in the solid that vanishes on the part of the boundary of the solid where essential boundary conditions ${ }^{\star}$ are prescribed, $\overline{\mathbf{v}}^{\mathrm{f}}$ the virtual velocity in the fluid that vanishes on the part of the boundary of the fluid where essential boundary conditions are specified, $\mathbf{a} \cdot \mathbf{b}$ the inner product between tensors $\mathbf{a}$ and $\mathbf{b}$ of the same order, and $\partial \mathbf{v}^{(\mathrm{s})} / \partial n$ is the directional derivative of $\mathbf{v}^{(\mathrm{s})}$ along the outward unit normal $\mathbf{n}$ to the boundary $\partial \Omega$ of $\Omega$. The effect of inertia forces is

\footnotetext{
^ For a boundary-value problem with field equations involving derivatives of order $2 m$, boundary
} conditions involving derivatives of order at most $(m-1)$ are called essential; others are called natural. 
included in the density of body forces. The physical meaning of $\boldsymbol{\Pi}^{(\mathrm{s})}$ and $\boldsymbol{\tau}^{(\mathrm{s})}$ can be described in a way similar to that done in different contexts in [20,23]. We note that the external action $\boldsymbol{\tau}^{(\mathrm{s})}$ can be regarded as the sum of two different contributions, the first one is a doubly normal double force, i.e., an external areal action which works on the rate of opening, $\left(\nabla \mathbf{v}^{(\mathrm{s})} \cdot \mathbf{n} \otimes \mathbf{n}\right)$, along the outward unit normal $\mathbf{n}$ of pores on the boundary, the other one is a tangential couple working on the vorticity of the apparent velocity of the solid; this nomenclature is due to Germain [23].* The areal action is also considered in the Cosserat model for granular materials (see, e.g., [21]) and in the present problem vanishes. However, the doubly normal double force plays an important role in the dilatancy phenomenon studied here. A motivation for considering higher-order stresses in the solid will be provided below. We note that capillary type forces in the fluid, discussed in the literature by second-order stresses (see, e.g., [14-16, 26]), have been neglected here to keep the analysis tractable. Whereas we have included in equation (4) the internal supplies $\mathbf{m}^{(\mathrm{f})}$ and $\mathbf{m}^{(\mathrm{s})}$ of the linear momentum, the internal supplies of the moment of momentum have been neglected. This is consistent with the assumption that the stress in the fluid is a hydrostatic pressure. The symmetry of $\mathbf{T}^{(\mathrm{s})}$ follows from equation (4) by setting

$$
\overline{\mathbf{v}}^{(\mathrm{f})}=\overline{\mathbf{v}}^{(\mathrm{s})}=\text { velocity field of a rigid body motion, }
$$

that is, from the objectivity of the left-hand side of (4) which also implies that the sum of $\mathbf{m}^{(\mathrm{f})}$ and $\mathbf{m}^{\text {(s) }}$ equals zero.

By using the divergence theorem and exploiting the fact that equation (4) must hold for all virtual velocities vanishing on the part of the boundary where essential boundary conditions are given, we obtain the following set of field equations and boundary conditions:

$$
\begin{array}{ll}
\operatorname{div}\left(\mathbf{T}^{(\mathrm{s})}-\operatorname{div} \boldsymbol{\Pi}^{(\mathrm{s})}\right)-\mathbf{m}^{(\mathrm{s})}+\mathbf{b}^{(\mathrm{s})}=\mathbf{0}, & \text { in } \Omega, \\
-\nabla p^{(\mathrm{f})}-\mathbf{m}^{(\mathrm{f})}+\mathbf{b}^{(\mathrm{f})}=\mathbf{0}, & \text { in } \Omega, \\
\mathbf{m}^{(\mathrm{s})}+\mathbf{m}^{(\mathrm{f})}=\mathbf{0}, & \text { in } \Omega, \\
\left(\mathbf{T}^{(\mathrm{s})}-\operatorname{div} \boldsymbol{\Pi}^{(\mathrm{s})}\right) \mathbf{n}-\operatorname{div}_{\mathrm{s}}\left(\boldsymbol{\Pi}^{(\mathrm{s})} \mathbf{n}\right)=\mathbf{t}^{(\mathrm{s})}, & \text { on } \partial_{1} \Omega, \\
\left(\boldsymbol{\Pi}^{(\mathrm{s})} \mathbf{n}\right) \mathbf{n}=\tau^{(\mathrm{s})}, & \text { on } \partial_{1} \Omega, \\
\mathbf{v}^{(\mathrm{s})}=\hat{\mathbf{v}}^{(\mathrm{s})}, & \text { on } \partial_{2} \Omega, \\
-p^{(\mathrm{f})} \mathbf{n}=\mathbf{t}^{(\mathrm{f})}, & \text { on } \partial_{3} \Omega, \\
\mathbf{v}^{(\mathrm{f})} \cdot \mathbf{n}=\hat{v}^{(\mathrm{f})}, & \text { on } \partial_{4} \Omega .
\end{array}
$$

Here $\operatorname{div}_{\mathrm{s}}$ is the surface divergence on $\partial \Omega$, and $\partial_{1} \Omega$ and $\partial_{2} \Omega$ are complementary parts of the boundary $\partial \Omega$ of $\Omega$, where natural and essential boundary conditions, respectively, are prescribed for the solid; a similar interpretation holds for $\partial_{3} \Omega$ and $\partial_{4} \Omega$ for the fluid.

\footnotetext{
$\star$ Here $\mathbf{a} \otimes \mathbf{b}$ is the tensor product between the $n$th order tensor $\mathbf{a}$ and the $m$ th order tensor $\mathbf{b}$ defined as $(\mathbf{a} \otimes \mathbf{b}) \mathbf{c}=(\mathbf{b} \cdot \mathbf{c}) \mathbf{a}$ for every $m$ th order tensor $\mathbf{c} ; \mathbf{A} \cdot \mathbf{B}=\operatorname{tr}\left(\mathbf{A B}^{\mathrm{T}}\right)$ for tensors $\mathbf{A}$ and $\mathbf{B}$.
} 


\subsection{CONSTITUTIVE RELATIONS}

The balance laws (5) and (6) are to be supplemented by constitutive relations; we express these in terms of the internal energy. We presume that the mixture is at a uniform temperature, the constituents are deformed quasistatically so that their kinetic energy can be neglected, and no energy is dissipated. The continuum model we use in order to describe deformations of a solid matrix saturated with a fluid must account for the strain energy associated with the gradient of the mass density of the solid. At the macroscopic level, the dependence of the strain energy on the gradient of deformation describes the effects of the opening of neighboring pores on the pore cluster which is modelled as a solid material point. We assume that the internal energy density can be split into two parts: a part that depends upon the "local" deformation of the solid and the fluid particles and another part that depends upon a "nonlocal" measure of deformation of the solid particles; the latter is taken to be proportional to $\left|\nabla \rho^{(\mathrm{s})}\right|^{2}$. Thus we write the balance of energy as

$$
\begin{aligned}
& \frac{\mathrm{d}}{\mathrm{d} t} \int_{\Omega} \rho\left[\epsilon\left(\rho^{(\mathrm{f})}, \mathbf{F}^{(\mathrm{s})}, \mathbf{X}^{(\mathrm{s})}\right)+\frac{\lambda_{\mathrm{s}}}{2 \rho}\left|\nabla \rho^{(\mathrm{s})}\right|^{2}\right] \mathrm{d} V \\
& =\int_{\Omega}\left(\mathbf{b}^{(\mathrm{s})} \cdot \mathbf{v}^{(\mathrm{s})}+\mathbf{b}^{(\mathrm{f})} \cdot \mathbf{v}^{(\mathrm{f})}\right) \mathrm{d} V \\
& \quad+\int_{\partial \Omega}\left(\mathbf{t}^{(\mathrm{s})} \cdot \mathbf{v}^{(\mathrm{s})}+\mathbf{t}^{(\mathrm{f})} \cdot \mathbf{v}^{(\mathrm{f})}+\boldsymbol{\tau}^{(\mathrm{s})} \cdot \frac{\partial \mathbf{v}^{(\mathrm{s})}}{\partial n}\right) \mathrm{d} A
\end{aligned}
$$

which because of (4) implies that

$$
\begin{aligned}
& \frac{\mathrm{d}}{\mathrm{d} t} \int_{\Omega} \rho\left[\epsilon\left(\rho^{(\mathrm{f})}, \mathbf{F}^{(\mathrm{s})}, \mathbf{X}^{(\mathrm{s})}\right)+\frac{\lambda_{\mathrm{s}}}{2 \rho}\left|\nabla \rho^{(\mathrm{s})}\right|^{2}\right] \mathrm{d} V \\
& =\int_{\Omega}\left(\mathbf{m}^{(\mathrm{s})} \cdot \mathbf{v}^{(\mathrm{s})}+\mathbf{m}^{(\mathrm{f})} \cdot \mathbf{v}^{(\mathrm{f})}+\mathbf{T}^{(\mathrm{s})} \cdot \nabla \mathbf{v}^{(\mathrm{s})}-p^{(\mathrm{f})} \operatorname{div} \mathbf{v}^{(\mathrm{f})}\right. \\
& \left.\quad+\boldsymbol{\Pi}^{(\mathrm{s})} \cdot \nabla \nabla \mathbf{v}^{(\mathrm{s})}\right) \mathrm{d} V .
\end{aligned}
$$

Here $\mathbf{F}^{(\mathrm{s})}=\operatorname{Grad} \mathbf{x}=\partial \mathbf{x} / \partial \mathbf{X}$ is the deformation gradient for the solid, $\lambda_{\mathrm{s}}>0$ is a material parameter with units of Newton $\mathrm{m}^{6} / \mathrm{kg}^{2}$, and $\mathrm{d} / \mathrm{d} t$ signifies the material time derivative following the mean motion of the mixture.

By using the Reynolds transport theorem the left hand side of equation (14) can be represented as a linear functional of the velocity field of the two constituents. Thus the following constitutive equations for the partial Cauchy stresses $\mathbf{T}^{(a)}$, the solid-fluid interaction forces $\mathbf{m}^{(a)}, a=\mathrm{s}, \mathrm{f}$, and the second-order stress $\boldsymbol{\Pi}^{(\mathrm{s})}$ associated with the solid constituent must hold:

$$
\begin{aligned}
& \mathbf{T}^{(\mathrm{s})}=\rho \frac{\partial \epsilon}{\partial \mathbf{F}^{(\mathrm{s})}} \mathbf{F}^{(\mathrm{s}) \mathrm{T}}-\lambda_{\mathrm{s}}\left[\frac{f^{\mathrm{ss}}}{2}\left(1+\xi^{(\mathrm{f})}\right) \mathbf{I}+\nabla \rho^{(\mathrm{s})} \otimes \nabla \rho^{(\mathrm{s})}\right], \\
& p^{(\mathrm{f})}=\rho \rho^{(\mathrm{f})} \frac{\partial \epsilon}{\partial \rho^{(\mathrm{f})}}-\frac{\lambda_{\mathrm{s}}}{2} f^{\mathrm{ss}} \xi^{(\mathrm{f})},
\end{aligned}
$$




$$
\begin{aligned}
\boldsymbol{\Pi}^{(\mathrm{s})}= & -\lambda_{\mathrm{s}} \rho^{(\mathrm{s})} \mathbf{I} \otimes \nabla \rho^{(\mathrm{s})}, \\
\mathbf{m}^{(\mathrm{s})}= & -\mathbf{m}^{(\mathrm{f})}=-\rho\left[\xi^{(\mathrm{f})}\left(\nabla \mathbf{F}^{(\mathrm{s})}\right)^{\mathrm{T}} \frac{\partial \epsilon}{\partial \mathbf{F}^{(\mathrm{s})}}+\xi^{(\mathrm{f})} \mathbf{F}^{(\mathrm{s})-\mathrm{T}} \frac{\partial \epsilon}{\partial \mathbf{X}^{(\mathrm{s})}}-\xi^{(\mathrm{s})} \frac{\partial \epsilon}{\partial \rho^{(\mathrm{f})}} \nabla \rho^{(\mathrm{f})}\right. \\
& \left.+\frac{\lambda_{\mathrm{s}}}{2 \rho} \nabla\left(\xi^{(\mathrm{f})} f^{\mathrm{ss}}\right)\right],
\end{aligned}
$$

where

$$
f^{\mathrm{ss}}=\nabla \rho^{(\mathrm{s})} \cdot \nabla \rho^{(\mathrm{s})},
$$

and $\xi^{(\mathrm{f})}$ is the mass fraction of the fluid phase. Note that the partial Cauchy stress tensor $\mathbf{T}^{(\mathrm{s})}$ is symmetric and $\partial \epsilon / \partial \mathbf{F}^{(\mathrm{s})}$ equals the partial first Piola-Kirchhoff stress tensor of the solid constituent. Equations (15)-(18) are derived in [34] where Germain's [23] arguments are used to obtain constitutive relations for a second gradient porous matrix filled with an ideal fluid. Equation (13) does not include all features of a general second gradient linear elastic matrix; only density gradients have been assumed to affect the internal potential energy and contributions of other components of the third order tensor, $\operatorname{Grad} \mathbf{F}^{(\mathrm{s})}$, have not been considered. Furthermore, we only analyze static deformations of the mixture. Thus Darcy-type drag forces are not modeled.

\subsection{SPLITTING OF EXTERNAL SURFACE TRACTIONS INTO PARTIAL TRACTIONS}

We consider problems for which $\mathbf{b}^{(\mathrm{s})}=\mathbf{b}^{(\mathrm{f})}=\mathbf{0}$, i.e., there are only external surface tractions. In a physical problem, total surface tractions are prescribed either on a part or on all of the boundary of the region $\Omega$. Here we require that these tractions be assigned on all of the boundary of the mixture in the current configuration. However, the solution of the boundary-value problem defined by equations (5)-(12) requires that the partial surface tractions be specified. In order to find the partial tractions we assume the existence of a potential function such that

$$
\begin{gathered}
\int_{\partial_{\Omega}}\left(\mathbf{t}^{(\mathrm{s})} \cdot \mathbf{v}^{(\mathrm{s})}+\mathbf{t}^{(\mathrm{f})} \cdot \mathbf{v}^{(\mathrm{f})}+\boldsymbol{\tau}^{(\mathrm{s})} \cdot \frac{\partial \mathbf{v}^{(\mathrm{s})}}{\partial n}\right) \mathrm{d} A \\
=\frac{\mathrm{d}}{\mathrm{d} t} \int_{\Omega} \psi^{\mathrm{ext}}\left(\mathbf{x}, \rho^{(\mathrm{s})}, \rho^{(\mathrm{f})}, \nabla \rho^{(\mathrm{s})}\right) \mathrm{d} V .
\end{gathered}
$$

The external surface tractions for which equation (20) holds are conservative. It is readily apparent that not all conservative surface tractions are characterized by equation (20). Here we consider only those surface tractions which satisfy equation (20) and $\psi^{\text {ext }}$ depends upon deformations of the solid only through $\rho^{(\mathrm{s})}$ and $\nabla \rho^{(\mathrm{s})}$. Equation (20) is dictated by the intended application of studying static deformations of an annular cylindrical porous region filled with an inviscid fluid. 
Requiring that equation (20) hold for all choices of the velocity field, we obtain

$$
\begin{aligned}
& \frac{\partial \psi^{\mathrm{ext}}}{\partial \rho^{(\mathrm{s})}}-\operatorname{div}\left(\frac{\partial \psi^{\mathrm{ext}}}{\partial \nabla \rho^{\mathrm{s})}}\right)=C^{\mathrm{s}}, \quad \text { in } \Omega, \\
& \frac{\partial \psi^{\mathrm{ext}}}{\partial \rho^{(\mathrm{f})}}=C^{\mathrm{f}}, \quad \text { in } \Omega, \\
& \mathbf{t}^{(\mathrm{s})}=\left[-\frac{\partial \psi^{\mathrm{ext}}}{\partial \rho^{(\mathrm{s})}} \rho^{(\mathrm{s})}+\xi^{(\mathrm{s})} \psi^{\mathrm{ext}}-\frac{\partial \psi^{\mathrm{ext}}}{\partial \nabla \rho^{(\mathrm{s})}} \cdot \nabla \rho^{(\mathrm{s})}+\operatorname{div}\left(\rho^{(\mathrm{s})} \frac{\partial \psi^{\mathrm{ext}}}{\partial \nabla \rho^{(\mathrm{s})}}\right)\right. \\
& \left.+\rho^{(\mathrm{s})}\left(\frac{\partial \psi^{\mathrm{ext}}}{\partial \nabla \rho^{(\mathrm{s})}} \cdot \mathbf{n}\right) \operatorname{tr}\left(\nabla^{\mathrm{s}} \mathbf{n}\right)-\left(\frac{\partial \psi^{\mathrm{ext}}}{\partial \nabla \rho^{(\mathrm{s})}} \cdot \mathbf{n}\right) \frac{\partial \rho^{(\mathrm{s})}}{\partial n}\right] \mathbf{n} \\
& +\rho^{(\mathrm{s})} \nabla^{\mathrm{s}}\left(\frac{\partial \psi^{\mathrm{ext}}}{\partial \nabla \rho^{(\mathrm{s})}} \cdot \mathbf{n}\right), \\
& \mathbf{t}^{(\mathrm{f})}=\left(-\frac{\partial \psi^{\mathrm{ext}}}{\left.\partial \rho^{(\mathrm{f})} \rho^{(\mathrm{f})}+\xi^{(\mathrm{f})} \psi^{\mathrm{ext}}\right) \mathbf{n},} \quad \text { on } \partial \Omega,\right. \\
& \tau^{(\mathrm{s})}=-\left(\rho^{(\mathrm{s})} \frac{\partial \psi^{\mathrm{ext}}}{\partial \nabla \rho^{(\mathrm{s})}} \cdot \mathbf{n}\right) \mathbf{n}, \quad \text { on } \partial \Omega,
\end{aligned}
$$

where $\nabla^{s}$ is the surface gradient on $\partial \Omega$. Equations (21) and (22) with $C^{\mathrm{s}}$ and $C^{\mathrm{f}}$ as constants are necessary conditions for the existence of a $\psi^{\text {ext }}$ for which $\mathbf{b}^{(\mathrm{s})}=$ $\mathbf{b}^{(\mathrm{f})}=\mathbf{0}$.

Once the constitutive relations for external actions are specified, which in our model is equivalent to specifying $\psi^{\text {ext }}$, the partial surface tractions can be expressed in terms of the total surface tractions; see [33] for details. The consideration of conservative external tractions limits the space of admissible surface tractions. The often used assumption characterizing the partial surface tractions in terms of the volume fraction of the constituents and the total surface tractions cannot be deduced from our work. It is because no state parameter in addition to the solid and the fluid placement maps has been introduced.

For a nonpolar medium whose response does not depend upon second-order displacement gradients, Batra [4] showed that surface tractions cannot depend upon $\partial \mathbf{u} / \partial n$ where $\mathbf{u}$ is the displacement of a point.

Results of this section can be summarized as follows: (i) a variational principle describing static deformations of a solid-fluid mixture is formulated, (ii) the Euler-Lagrange equations are deduced from the principle, and are recognized as the balance laws and the constitutive relations for the solid-fluid mixture, (iii) the external and internal actions are specified by requiring that they be conservative.

\section{Solution of a Boundary-Value Problem}

We analyze, within a linearized second gradient theory, static infinitesimal deformations of a long hollow porous cylinder filled with an inviscid fluid and with the inner and the outer surfaces subjected to uniform external pressures $p_{1}^{\text {ext }}$ and $p_{2}^{\text {ext }}$, 
respectively. We assume that the pressure on the inner and the outer surfaces of the cylinder, in the reference configuration, equals $p_{01}^{\text {ext }}$ and $p_{02}^{\text {ext }}$, respectively, and postulate that

$$
\begin{aligned}
\epsilon= & \frac{1}{\rho_{0}}\left[\mathbf{T}^{0(\mathrm{~s})} \cdot \mathbf{H}^{(\mathrm{s})}+\gamma^{0 \mathrm{f}} \Delta \rho^{(\mathrm{f})}+\frac{1}{2} \mathbb{C}\left[\mathbf{H}^{(\mathrm{s})}\right] \cdot \mathbf{H}^{(\mathrm{s})}-\frac{1}{4} \mathbf{H}^{(\mathrm{s})} \cdot\left(\mathbf{T}^{0(\mathrm{~s})} \mathbf{H}^{(\mathrm{s}) \mathrm{T}}\right.\right. \\
& \left.-\mathbf{H}^{(\mathrm{s})} \mathbf{T}^{0(\mathrm{~s})}\right)+\frac{1}{8} \mathbf{H}^{(\mathrm{s})} \cdot\left(\mathbf{H}^{(\mathrm{s})} \mathbf{T}^{0(\mathrm{~s})}-\mathbf{T}^{0(\mathrm{~s})} \mathbf{H}^{(\mathrm{s})}+\mathbf{T}^{0(\mathrm{~s})} \mathbf{H}^{(\mathrm{s}) \mathrm{T}}-\mathbf{H}^{(\mathrm{s}) \mathrm{T}} \mathbf{T}^{0(\mathrm{~s})}\right) \\
& \left.+\frac{1}{2} \gamma^{\mathrm{ff}}\left(\Delta \rho^{(\mathrm{f})}\right)^{2}+\Delta \rho^{(\mathrm{f})} \mathbf{K}^{\mathrm{sf}} \cdot \mathbf{H}^{(\mathrm{s})}\right]
\end{aligned}
$$

where

$$
\mathbf{H}^{(\mathrm{s})}:=\nabla \mathbf{u}^{(\mathrm{s})}, \quad \Delta \rho^{(\mathrm{f})}=\rho^{(\mathrm{f})}-\rho^{0(\mathrm{f})},
$$

$\rho^{0(\mathrm{f})}$ is the mass density of the fluid in the reference configuration, $\mathbf{T}^{0(\mathrm{~s})}$ a symmetric tensor representing the partial stress in the solid in the reference configuration, $\mathbb{C}$ is the classical elasticity tensor for the solid constituent mapping symmetric second order tensors into symmetric second order tensors, $\gamma^{0 \mathrm{ff}}, \gamma^{\mathrm{ff}}$ and $\mathbf{K}^{\text {sf }}=\mathbf{K}^{\text {sfT }}$ are material parameters.

Terms involving the second order tensor $\mathbf{T}^{(\mathrm{s})}$ and the scalar $\gamma^{(0) \mathrm{f}}$ in equation (26) represent contributions to the internal potential energy by the prestress in the solid and the fluid constituents. Since a fluid can be in equilibrium only if it is confined, it is necessary to consider a pre-stressed reference configuration. Equation (26) is the most general one can have to get linear constitutive relations for a pre-stressed solid-fluid mixture. Equations (15)-(18) imply that terms of order one in $\mathbf{H}^{(\mathrm{s})}$ and $\rho^{(\mathrm{f})}$ yield zeroth order terms for the solid stress tensor and the fluid pressure, and the second-order terms in $\mathbf{H}^{(\mathrm{s})}$ and $\rho^{(\mathrm{f})}$ provide the first-order terms for the solid stress tensor, the fluid pressure, and the bulk internal action $\mathbf{m}^{(\mathrm{s})}$.

The second-order tensor $\mathbf{K}^{\text {sf }}$ accounts for the interaction between the solid and the fluid phases because of deformations of pores; $\mathbf{K}^{\text {sf }}$ is not necessarily a spherical tensor even when the solid and the fluid constituents are isotropic. It is because pores need not be of uniform shapes and sizes.

The coupling coefficient $\mathbf{K}^{\text {sf }}$ can be explained in terms of the pore dilatancy as follows: the dilatation of pores induced by the injection of the solid or the fluid into a suitable elementary reference volume either deforms the solid constituent or changes the mass density of the fluid or both.

Denoting the first term on the right-hand side of equation (15) by $\overline{\mathbf{T}}^{(\mathrm{s})}$ and using (26) we obtain

$$
\begin{aligned}
\overline{\mathbf{T}}^{(\mathrm{s})}= & \mathbf{T}^{0(\mathrm{~s})}+\frac{\Delta \rho^{(\mathrm{f})}}{\rho_{0}} \mathbf{T}^{0(\mathrm{~s})}-\xi_{0}^{(\mathrm{s})} \operatorname{tr}\left(\mathbf{H}^{(\mathrm{s})}\right) \mathbf{T}^{0(\mathrm{~s})}+\frac{1}{2}\left(\mathbf{T}^{0(\mathrm{~s})} \mathbf{H}^{(\mathrm{s}) \mathrm{T}}+\mathbf{H}^{(\mathrm{s})} \mathbf{T}^{0(\mathrm{~s}) \mathrm{T}}\right) \\
& +\frac{1}{2}\left(\mathbf{W}^{(\mathrm{s})} \mathbf{T}^{0(\mathrm{~s})}-\mathbf{T}^{0(\mathrm{~s})} \mathbf{W}^{(\mathrm{s})}\right)+\mathbb{C}\left[\mathbf{E}^{(\mathrm{s})}\right]+\Delta \rho^{(\mathrm{f})} \mathbf{K}^{\mathrm{sf}},
\end{aligned}
$$


where $\mathbf{E}^{(\mathrm{s})}:=\left(\mathbf{H}^{(\mathrm{s})}+\mathbf{H}^{(\mathrm{s}) \mathrm{T}}\right) / 2$ and $\mathbf{W}^{(\mathrm{s})}:=\left(\mathbf{H}^{(\mathrm{s})}-\mathbf{H}^{(\mathrm{s}) \mathrm{T}}\right) / 2$. If each component of the initial stress, $\mathbf{T}^{0(\mathrm{~s})}$, is much less than the smallest value of an elastic modulus of the solid phase, then terms such as $\mathbf{T}^{\text {(s) }} \mathbf{H}^{(\mathrm{s}) \mathrm{T}}$ can be neglected in equation (28). We assume the following form for the elasticity tensor applied to the $\mathbf{E}^{(\mathrm{s})}$

$$
\mathbb{C}\left[\mathbf{E}^{(\mathrm{s})}\right]=\lambda \operatorname{tr}\left(\mathbf{E}^{(\mathrm{s})}\right) \mathbf{I}+2 \mu \mathbf{E}^{(\mathrm{s})},
$$

where $\lambda$ and $\mu$ are the Lamé constants for the solid. One can deduce expressions for $p^{(\mathrm{f})}$ and $\mathbf{m}^{(\mathrm{s})}$ by substituting for $\epsilon$ from (26) into (16) and (18).

We assume that deformations of the mixture are plane strain, and in the plane of deformation they are independent of the angular position. That is, in cylindrical coordinates the two in-plane physical components $u_{r}$ and $u_{\theta}$ of the displacement are functions of the radial coordinate $r$ only and $u_{z}=0$; we call such a deformation field radially symmetric. This assumption is reasonable because the body and the boundary conditions are radially symmetric. Henceforth we use cylindrical coordinates with orthonormal vectors $\mathbf{e}_{r}$ and $\mathbf{e}_{\theta}$ at a point in the radial and the circumferential directions, respectively, and work in terms of physical components of stresses. In order to find boundary conditions on the solid and the fluid phases, we consider external tractions given by the following expression for $\psi^{\text {ext }}$ :

$$
\psi^{\mathrm{ext}}=C^{\mathrm{s}} \rho^{(\mathrm{s})}+C^{\mathrm{f}} \rho^{(\mathrm{f})}+\mathbf{C}^{\mathrm{int}}(r, \theta) \cdot \nabla \rho^{(\mathrm{s})} \Delta \rho^{(\mathrm{s})}+p_{0}+p_{1} r .
$$

Here $\Delta \rho^{(\mathrm{s})}=\rho^{(\mathrm{s})}-\rho^{0(\mathrm{~s})}, \rho^{0(\mathrm{~s})}$ is the density of the solid phase in the reference configuration, $\mathbf{C}^{\text {int }}(r, \theta)$ is a solenoidal vector field whose radial component is independent of $r$ and $\theta$. It is easy to verify that the aforementioned assumptions on $\mathbf{C}^{\text {int }}(r, \theta)$ suffice to satisfy equation (21). Bearing in mind the hypothesis of radially symmetric deformations for both phases, we have

$$
\mathbf{C}^{\text {int }}(r, \theta) \cdot \nabla \rho^{(\mathrm{s})} \Delta \rho^{(\mathrm{s})}=\mathbf{C}^{\text {int }}(r, \theta) \cdot\left(\rho^{(\mathrm{s})^{\prime}} \mathbf{e}_{r}\right) \Delta \rho^{(\mathrm{s})}=C^{\text {int }} \rho^{(\mathrm{s})^{\prime}} \Delta \rho^{(\mathrm{s})},
$$

where $C^{\text {int }}$ is the constant radial component of $\mathbf{C}^{\text {int }}(r, \theta)$ and a prime indicates differentiation with respect to $r$. Substitution from (30) and (31) into equations (23)(25) gives

$$
\begin{aligned}
& \mathbf{t}^{(\mathrm{s})}=\left[\xi^{(\mathrm{s})} \psi^{\mathrm{ext}}-C^{\mathrm{s}} \rho^{(\mathrm{s})}-C^{\mathrm{int}} \Delta \rho^{(\mathrm{s})} \rho^{(\mathrm{s})^{\prime}}+\frac{C^{\mathrm{int}}}{r} \rho^{(\mathrm{s})} \Delta \rho^{(\mathrm{s})}\right] \mathbf{n}, \\
& \mathbf{t}^{(\mathrm{f})}=\left[\xi^{(\mathrm{f})} \psi^{\mathrm{ext}}-C^{\mathrm{f}} \rho^{(\mathrm{f})}\right] \mathbf{n}, \\
& \boldsymbol{\tau}^{(\mathrm{s})}=-\left(\rho^{(\mathrm{s})} \Delta \rho^{(\mathrm{s})} C^{\mathrm{int}} \delta\right) \mathbf{n},
\end{aligned}
$$

where $\delta=+1$ on the external surface and $\delta=-1$ on the internal surface. Note that values of $C^{\mathrm{s}}, C^{\mathrm{f}}$ and $C^{\mathrm{int}}$ depend upon the shape of the bounding surface, constituents of the mixture, and on the interaction between the mixture and the medium surrounding it. For example, at an impermeable wall, $C^{\text {int }}=0$ because externally applied tractions of type (34) vanish; as a matter of fact, the absence of fluid flux implies that the fluid belonging to the cluster of pores near the boundary 
rests at a uniform pressure. In other words the external world cannot access pores within the body and dilate them.

Because of our interest in studying infinitesimal deformations under the action of higher-order tractions (or double forces without moments), we retain terms in (32)-(34) that are bilinear in $\Delta \rho^{(\mathrm{s})}$ and $\rho^{(\mathrm{s})^{\prime}}$; the remaining terms are either linear in $\rho^{(\mathrm{s})}$ and $\rho^{(\mathrm{f})}$ or independent of $\rho^{(\mathrm{s})}$ and $\rho^{(\mathrm{f})}$. We thus get the following linearized constitutive characterizations of external tractions and double forces: ${ }^{\star}$

$$
\begin{aligned}
\mathbf{t}^{(\mathrm{s})}= & {\left[-\xi^{0(\mathrm{f})} \xi^{0(\mathrm{~s})} \rho^{0}\left(C^{(\mathrm{s})}-C^{(\mathrm{f})}\right)+\xi^{0(\mathrm{~s})} \bar{p}_{0}\right] \mathbf{n} } \\
& +\left[\left(-\left(\xi^{0(\mathrm{f})}\right)^{2}\left(C^{(\mathrm{s})}-C^{(\mathrm{f})}\right)+\xi^{0(\mathrm{f})} \frac{\bar{p}_{0}}{\rho^{0}}+\frac{C^{\mathrm{int}}}{r} \rho^{0(\mathrm{~s})}\right) \Delta \rho^{(\mathrm{s})}\right. \\
& \left.+\left(-\left(\xi^{0(\mathrm{~s})}\right)^{2}\left(C^{(\mathrm{s})}-C^{(\mathrm{f})}\right)-\xi^{0(\mathrm{~s})} \frac{\bar{p}_{0}}{\rho^{0}}\right) \Delta \rho^{(\mathrm{f})}+\xi^{0(\mathrm{~s})}\left(\tilde{p}_{0}+r p_{1}\right)\right] \mathbf{n}, \\
\mathbf{t}^{(\mathrm{f})}= & {\left[\xi^{0(\mathrm{f})} \xi^{0(\mathrm{~s})} \rho^{0}\left(C^{(\mathrm{s})}-C^{(\mathrm{f})}\right)+\xi^{0(\mathrm{f})} \bar{p}_{0}\right] \mathbf{n} } \\
& +\left[\left(\left(\xi^{0(\mathrm{~s})}\right)^{2}\left(C^{(\mathrm{s})}-C^{(\mathrm{f})}\right)+\xi^{0(\mathrm{~s})} \frac{\bar{p}_{0}}{\rho^{0}}\right) \Delta \rho^{(\mathrm{f})}\right. \\
& \left.+\left(\left(\xi^{0(\mathrm{f})}\right)^{2}\left(C^{(\mathrm{s})}-C^{(\mathrm{f})}\right)-\xi^{0(\mathrm{f})} \frac{\bar{p}_{0}}{\rho^{0}}\right) \Delta \rho^{(\mathrm{s})}+\xi^{0(\mathrm{f})}\left(\tilde{p}_{0}+r p_{1}\right)\right] \mathbf{n}, \\
\boldsymbol{\tau}^{(\mathrm{s})}= & -\rho^{0(\mathrm{~s})} C^{\mathrm{int}} \Delta \rho^{(\mathrm{s})} \delta \mathbf{n} .
\end{aligned}
$$

Here $\xi^{0(\mathrm{f})}$ and $\xi^{0(\mathrm{~s})}$ are mass fractions of both phases in the initial configuration, $\rho^{0}$ is the apparent density of the mixture in this configuration, $\bar{p}_{0}$ is the reference value of $p_{0}$ and $p_{1}$ equals its infinitesimal variation. Note that terms in the first brackets in the representation formulas of $\mathbf{t}^{(\mathrm{s})}$ and $\mathbf{t}^{(\mathrm{f})}$ describe tractions applied on the boundary of the mixture in the reference configuration, and terms in the second brackets are infinitesimal increments of tractions. Similarly equation (37) states that the initial double force vanishes but its infinitesimal increment is nonzero.

Let $R_{1}$ and $R_{2}$ denote the inner and the outer radii of the porous cylinder in the reference configuration. It is clear from equation (28) that $\mathbf{T}^{0(\mathrm{~s})}$ is the partial stress in the solid in the reference configuration. Boundary conditions for the solid in the reference configuration are

$$
\begin{aligned}
& \mathbf{T}^{0(\mathrm{~s})}\left(R_{1}\right) \mathbf{n}=\mathbf{t}^{(\mathrm{s})}\left(R_{1}\right)=-d_{01}^{(\mathrm{s})} p_{01}^{\mathrm{ext}} \mathbf{n}, \\
& \mathbf{T}^{0(\mathrm{~s})}\left(R_{2}\right) \mathbf{n}=\mathbf{t}^{(\mathrm{s})}\left(R_{2}\right)=-d_{02}^{(\mathrm{s})} p_{02}^{\mathrm{ext}} \mathbf{n},
\end{aligned}
$$

where $d_{01}^{(\mathrm{s})}$ and $d_{02}^{(\mathrm{s})}$ denote fractions of the externally applied pressures, $p_{01}^{\text {ext }}$ and $p_{02}^{\text {ext }}$, carried by the solid phase on the inner and the outer surfaces of the porous

\footnotetext{
^ We have assumed that the small parameter defining the linearization procedure is same for the solid and the fluid kinematical descriptors. Thus small deformations of the solid matrix are associated with small variations of the fluid apparent density.
} 
cylindrical body. Through these equations a representation formula for both areal fractions splitting external pressure into partial tractions is obtained. Let the state of stress, $\mathbf{T}^{0 \text { (s) }}$ and $p_{0}^{(\mathrm{f})}$, be

$$
\begin{aligned}
& \mathbf{T}^{0(\mathrm{~s})}=\left(a_{0}-\frac{b_{0}}{r^{2}}\right) \mathbf{e}_{r} \otimes \mathbf{e}_{r}+\left(a_{0}+\frac{b_{0}}{r^{2}}\right) \mathbf{e}_{\theta} \otimes \mathbf{e}_{\theta}, \\
& p_{0}^{(\mathrm{f})}:=\gamma^{0 \mathrm{f}} \rho^{0(\mathrm{f})}=\mathrm{const}=\left(1-d_{01}^{(\mathrm{s})}\right) p_{01}^{\mathrm{ext}}=\left(1-d_{02}^{(\mathrm{s})}\right) p_{02}^{\mathrm{ext}},
\end{aligned}
$$

then

$$
\begin{aligned}
& a_{0}=-\frac{R_{2}^{2} d_{02}^{(\mathrm{s})} p_{02}^{\mathrm{ext}}-R_{1}^{2} d_{01}^{(\mathrm{s})} p_{01}^{\mathrm{ext}}}{R_{2}^{2}-R_{1}^{2}} \\
& b_{0}=-\frac{R_{1}^{2} R_{2}^{2}\left(d_{02}^{\mathrm{s})} p_{02}^{\mathrm{ext}}-d_{01}^{\mathrm{s})} p_{01}^{\mathrm{ext}}\right)}{R_{2}^{2}-R_{1}^{2}} .
\end{aligned}
$$

It is easily verified (see, e.g., [32]) that the pre-stress (40) is admissible. Equation (40) 2 implies that $d_{01}^{(\mathrm{s})}, d_{02}^{(\mathrm{s})}, p_{01}^{\text {ext }}$ and $p_{02}^{\text {ext }}$ cannot be independently prescribed. For example, if $d_{01}^{(\mathrm{s})}=d_{02}^{(\mathrm{s})}$ for a reference configuration, then $p_{01}^{\text {ext }}$ must equal $p_{02}^{\text {ext }}$, and the stress state in the solid phase must also be that of a hydrostatic pressure, as $b_{0}=0$ (see equation (41)). At an impermeable wall, $d_{01}^{\text {(s) }}$ need not equal 1 because a part of external tractions applied to the impermeable wall may be carried by the fluid.

Equations for the determination of infinitesimal displacements in the radial and in the circumferential directions and for $\Delta \rho^{(\mathrm{f})}$ are

$$
\begin{aligned}
& -\lambda_{\mathrm{s}} \rho^{0(\mathrm{~s})^{2}}\left(u_{r}^{\prime \prime \prime \prime}+\frac{2}{r} u_{r}^{\prime \prime \prime}-\frac{3}{r^{2}} u_{r}^{\prime \prime}+\frac{3}{r^{3}} u_{r}^{\prime}-\frac{3}{r^{4}} u_{r}\right) \\
& +\left[2 \xi^{0(\mathrm{f})}\left(a_{0}-\frac{b_{0}}{r^{2}}\right)+\lambda+2 \mu\right] u_{r}^{\prime \prime} \\
& +\frac{1}{r}\left[2 \xi^{0(\mathrm{f})}\left(a_{0}+\frac{b_{0}}{r^{2}}\right)+\frac{2 b_{0}}{r^{2}}+\lambda+2 \mu\right] u_{r}^{\prime} \\
& +\frac{1}{r^{2}}\left[-2 \xi^{0(\mathrm{f})}\left(a_{0}+\frac{b_{0}}{r^{2}}\right)-\frac{2 b_{0}}{r^{2}}-(\lambda+2 \mu)\right] u_{r} \\
& +\left[\frac{1}{\rho_{0}}\left(a_{0}-\frac{b_{0}}{r^{2}}\right)+K_{r r}^{\mathrm{sf}}-\xi^{0(\mathrm{~s})} \gamma^{0(\mathrm{f})}\right] \Delta \rho^{(\mathrm{f})^{\prime}} \\
& +\frac{1}{r}\left[K_{r r}^{\mathrm{sf}}-K_{\theta \theta}^{\mathrm{sf}}\right] \Delta \rho^{(\mathrm{f})}=0, \\
& \frac{1}{r^{2}}\left[\left(\frac{a_{0}}{2}+\mu\right) r^{2}-b_{0}\right] u_{\theta}^{\prime \prime}+\frac{1}{r^{3}}\left[\left(\frac{a_{0}}{2}+\mu\right) r^{2}-b_{0}\right] u_{\theta}^{\prime} \\
& \quad-\frac{1}{r^{4}}\left[\left(\frac{a_{0}}{2}+\mu\right) r^{2}-b_{0}\right] u_{\theta}+K_{r \theta}^{\mathrm{sf}}\left(\Delta \rho^{(\mathrm{f})^{\prime}}+\frac{2}{r} \Delta \rho^{(\mathrm{f})}\right)=0,
\end{aligned}
$$




$$
\begin{aligned}
& \left(2 \rho^{0(\mathrm{f})} \gamma^{0 \mathrm{f}}+\rho^{0} \rho^{0(\mathrm{f})} \gamma^{\mathrm{ff}}\right) \Delta \rho^{(\mathrm{f})} \\
& =c+\rho^{0(\mathrm{~s})} \rho^{0(\mathrm{f})} \gamma^{0 \mathrm{f}}\left(u_{r}^{\prime}+\frac{1}{r} u_{r}\right) \\
& \quad-\rho^{0} \rho^{0(\mathrm{f})}\left[K_{r r}^{\mathrm{sf}} u_{r}^{\prime}+K_{r \theta}^{\mathrm{sf}}\left(u_{\theta}^{\prime}-\frac{1}{r} u_{\theta}\right)+K_{\theta \theta}^{\mathrm{sf}} \frac{1}{r} u_{r}\right] \\
& \quad-\rho^{0(\mathrm{f})}\left(a_{0}-\frac{b_{0}}{r^{2}}\right) u_{r}^{\prime}-\rho^{0(\mathrm{f})}\left(a_{0}+\frac{b_{0}}{r^{2}}\right) \frac{1}{r} u_{r} .
\end{aligned}
$$

In equation (44), $c$ is a constant of integration. Equation (44) can be solved for $\Delta \rho^{(\mathrm{f})}$ and the result substituted into equations (42) and (43) to obtain two coupled ordinary differential equations for $u_{r}$ and $u_{\theta}$. These equations belong to Heun's family of equations (see [3]); their solution has four poles, one at $r=0$, one at $r=\infty$, and locations of the other two poles depend upon the coefficient of $u_{\theta}^{\prime \prime}$ in equation (43). In order for a pole to be within the hollow cylinder the following inequalities must hold

$$
R_{1}<\sqrt{\frac{b_{0}}{a_{0} / 2+\mu}}<R_{2} .
$$

Thus depending upon the shear modulus of the solid phase, the pre-stress, and the fraction of externally applied pressures carried by the solid constituent, the solution may blow up at a point within the annular cylinder.

\subsection{A SIMPLIFIED PROBLEM}

Henceforth we consider the case of $b_{0}=0$, therefore $r=0$ is a triple pole and there is no pole within the hollow cylinder. The stress in the solid phase in the reference configuration is a hydrostatic pressure. Thus $d_{02}^{\text {(s) }} p_{02}^{\text {ext }}=d_{01}^{\text {(s) }} p_{01}^{\text {ext }}=-a_{0}$, i.e., $-a_{0}$ equals the reference value of the hydrostatic pressure in the solid. Moreover $\bar{p}_{0}$ equals the negative of the external pressure applied on both the external and the internal surfaces of the hollow cylinder

$$
p_{01}^{\mathrm{ext}}=p_{02}^{\mathrm{ext}}=-\bar{p}_{0}
$$

We also assume that the second order tensor $\mathbf{K}^{\text {sf }}$ is spherical: $\mathbf{K}^{\text {sf }}=K^{\text {sf }} \mathbf{I}$. Thus the strain-energy density $\epsilon$ provides both the dependence of the hydrostatic component of the solid partial stress tensor on $\Delta \rho^{(\mathrm{f})}$, and that of the hydrostatic pressure acting in the fluid on $\Delta \rho^{(\mathrm{s})}$. An increment $\Delta \rho^{(\mathrm{f})}$ in the apparent mass density of the fluid does not induce nonzero deviatoric stresses in the solid constituent.

\subsubsection{Influence of the Coupling Coefficient $K^{\text {sf }}$ on Density Profiles}

With the aforestated assumptions and the existence of a first integral of equation (42), equations (42)-(44) can be reduced to the following two uncoupled 
ordinary differential equations:

$$
\begin{aligned}
& -\lambda_{\mathrm{s}} \rho^{0(\mathrm{~s})^{2}}\left[U_{r}^{\prime \prime}+\frac{1}{r} U_{r}^{\prime}\right] \\
& +\left[2 \mu+\lambda+2 \xi^{0(\mathrm{f})} a_{0}-\frac{\left(\xi^{0(\mathrm{~s})} \gamma^{0(\mathrm{f})}-a_{0} / \rho^{0}-K^{\mathrm{sf}}\right)^{2}}{2 \gamma^{0(\mathrm{f})} / \rho^{0}+\gamma^{\mathrm{ff}}}\right] U_{r}=\Gamma_{\mathrm{s}}, \\
& U_{\theta}^{\prime}+\frac{2}{r} U_{\theta}=0,
\end{aligned}
$$

where $\Gamma_{\mathrm{s}}$ is an integration constant to be determined by boundary conditions, $U_{r}=$ $\left(u_{r}^{\prime}+(1 / r) u_{r}\right)=\operatorname{tr} \mathbf{H}^{(\mathrm{s})}$ and $U_{\theta}=\left(u_{\theta}^{\prime}-(1 / r) u_{\theta}\right)$ are dimensionless quantities. In particular, $U_{r}$ is related to the increment of the solid apparent density by

$$
\Delta \rho^{(\mathrm{s})}=-\rho^{0(\mathrm{~s})} U_{r} .
$$

According to the previous assumptions we can also specify boundary conditions arising from equations (9)-(12) and equations (35)-(37). Recalling the structure of balance laws (5) and (6) these conditions prescribe external radial and circumferential tractions for the solid constituent acting on the inner and the outer surfaces, the external pressure for the fluid constituent acting on the inner (or the outer) surface and the double forces only for the solid constituent acting on disjoint parts of the boundary. The differential equation for the variable $U_{r}$ is a Bessel equation, and that for $U_{\theta}$ is the classical Euler equation.

Let us first consider equation (48) and boundary conditions corresponding to shear external tractions for the solid constituent. As these tractions are null, see equation (35), we get

$$
U_{\theta}=0 \quad \Rightarrow \quad u_{\theta}=c_{\theta} r
$$

$c_{\theta}$ being a constant of integration. Thus the only admissible infinitesimal displacement in the circumferential direction is a rigid body rotation.

In order to solve equation (47), we recall that a typical Bessel equation is

$$
x^{2} y^{\prime \prime}(x)+x y^{\prime}(x)+\left(x^{2}-v^{2}\right) y(x)=0,
$$

and a typical modified Bessel equation is

$$
x^{2} y^{\prime \prime}(x)+x y^{\prime}(x)-\left(x^{2}+v^{2}\right) y(x)=0 .
$$

It is evident that equation (47) for $\Gamma_{\mathrm{s}}=0$ is either a classical or a modified Bessel equation, according to the sign of the coefficient of $U_{r}$. Therefore, two different solutions for the increment of the solid apparent density can be obtained. Let the coefficient of $U_{r}$ in equation (47) be defined by

$$
q:=2 \mu+\lambda+2 \xi^{0(\mathrm{f})} a_{0}-\frac{\left(\xi^{0(\mathrm{~s})} \gamma^{0(\mathrm{f})}-a_{0} / \rho^{0}-K^{\mathrm{sf}}\right)^{2}}{2 \gamma^{0(\mathrm{f})} / \rho^{0}+\gamma^{\mathrm{ff}}},
$$


then, through a change of variable, equation (47) for $\Gamma_{\mathrm{s}}=0$ becomes

$$
\frac{\mathrm{d}^{2} U_{r}(\xi)}{\mathrm{d} \xi^{2}}+\frac{1}{\xi} \frac{\mathrm{d} U_{r}(\xi)}{\mathrm{d} \xi}-\operatorname{sign}(q) U_{r}(\xi)=0, \quad \xi=\sqrt{\frac{|q|}{\lambda_{\mathrm{s}} \rho^{0(\mathrm{~s})^{2}}}} r .
$$

Depending upon the sign of $q$, equation (52) is either a Bessel or a modified Bessel equation. If $q>0$ then the solution of equation (52) is given by a linear combination of modified Bessel functions $I_{0}(\xi)$ and $K_{0}(\xi)$. However, if $q<0$ then the solution of equation (52) is given by a linear combination of classical Bessel functions $J_{0}(\xi)$ and $Y_{0}(\xi)$. The subscript zero indicates that these functions are solutions of the Bessel equation with $v=0$. Note that the standard nomenclature for Bessel equations has been adopted. Expressions for the classical Bessel functions $J_{0}(\xi)$ and $Y_{0}(\xi)$ and the modified Bessel functions $I_{0}(\xi)$ and $K_{0}(\xi)$ can be found in a book, e.g., [1].

Starting from a vanishing coupling coefficient we conduct a parametric study of the solution of equation (47) when $\left|K^{\text {sf }}\right|$ is monotonically increased. This is essentially necessitated by our inability to identify the suitable range of values of $K^{\text {sf }}$. Even though we know that it describes the effects of pore dilatancy, there is no experimental data available to quantify this effect. We commence from the solution of the homogeneous equation associated with equation (47). Two different ranges of values of the coupling coefficient can be determined. Let

$$
K_{1,2}^{\mathrm{sf}}:=\xi^{0(\mathrm{~s})} \gamma^{0(\mathrm{f})}-\frac{a_{0}}{\rho^{0}} \mp \sqrt{\left(2 \mu+\lambda+2 \xi^{0(\mathrm{f})} a_{0}\right)\left(\frac{2 \gamma^{0(\mathrm{f})}}{\rho^{0}}+\gamma^{\mathrm{ff}}\right)}
$$

be the values of $K^{\text {sf }}$ for which $q$ vanishes. When $K^{\text {sf }} \in\left(K_{1}^{\text {sf }}, K_{2}^{\text {sf }}\right)$ then the solution of the homogeneous equation is given by a linear combination of the modified Bessel functions as $\operatorname{sign}(q)=1$. For $K^{\text {sf }} \in\left(-\infty, K_{1}^{\text {sf }}\right) \cup\left(K_{2}^{\text {sf }}, \infty\right)$ then the solution of the aforementioned equation is a linear combination of the classical Bessel functions as $\operatorname{sign}(q)=-1$.

Once equation (52) has been solved, the solution of equation (47) is obtained by simply adding a suitable constant to it. The following figures depict the $\Delta \rho^{(\mathrm{s})}$ profiles for a salt matrix filled with brine; values of constitutive and geometric parameters and surface tractions applied on the boundary are listed in Table I. Values of constitutive parameters are deduced from the test data on salt rock and brine [37]. Note that values for the constitutive coefficients $\lambda_{\mathrm{s}}$ and $C^{\text {int }}$ that describe second gradient effects are arbitrarily chosen since no experimental data is available for their determination. However, they are reasonable and can describe the pore-opening effect near the boundary of the mixture. The variation of $\Delta \rho^{(\mathrm{s})}$ in the boundary layer due to second gradient is about $1 \%$ of its reference value (i.e. its value due to the first gradient model only). The plot in Figure 1 is representative of solutions in the range $\left(K_{1}^{\mathrm{sf}}, K_{2}^{\mathrm{sf}}\right)$ and that in Figure 2 corresponds to solutions in the range $\left(-\infty, K_{1}^{\text {sf }}\right) \cup\left(K_{2}^{\text {sf }}, \infty\right)$. In Figure 1 the typical behavior of fields exhibiting boundary layers is shown; in our model these boundary layers are driven by the 
Table I. Values of parameters used in the computation of results. $E$ and $v$ are Young's modulus and Poisson's ratio of the solid matrix; $\hat{\rho}^{0(\mathrm{~s})}$ and $\hat{\rho}^{0(\mathrm{f})}$ are the densities of the solid and the fluid constituent in the reference configuration, $v^{0(\mathrm{~s})}$ and $v^{0(\mathrm{f})}$ their volume fractions. In the reference configuration the mixture is saturated

\begin{tabular}{lll}
\hline Constitutive parameters & Geometric and referencial state properties & \multicolumn{1}{c}{ Tractions } \\
\hline$E=200 \mathrm{MPa}$ & $\hat{\rho}^{0(\mathrm{~s})}=1850 \mathrm{~kg} / \mathrm{m}^{3}$ & $p_{01}^{\text {ext }}=20 \mathrm{MPa}$ \\
$\nu=0.33$ & $\hat{\rho}^{0(\mathrm{f})}=1300 \mathrm{~kg} / \mathrm{m}^{3}$ & $p_{02}^{\text {ext }}=p_{01}^{\text {ext }}$ \\
$\lambda_{\mathrm{s}}=200 \mathrm{~N} \mathrm{~m}^{4} / \mathrm{kg}^{2}$ & $v^{0(\mathrm{~s})}=0.97$ & $\tilde{p}_{0}=-2.21 \mathrm{MPa}$ \\
$\gamma_{\mathrm{ff}}=1.6410^{6} \mathrm{~N} \mathrm{~m}^{4} / \mathrm{kg}^{2}$ & $v^{0(\mathrm{f})}=1-v^{0(\mathrm{~s})}=0.03$ & $p_{1}=0.1 \cdot 10^{6} \mathrm{~N} / \mathrm{m}^{3}$ \\
$C^{(\mathrm{s})}=C^{(\mathrm{f})}$ & $\rho^{0(\mathrm{~s})}=\hat{\rho}^{0(\mathrm{~s})} v^{0(\mathrm{~s})}=1794.5 \mathrm{~kg} / \mathrm{m}^{3}$ & \\
$C^{\text {int }}=1 \mathrm{~N} \mathrm{~m}^{3} / \mathrm{kg}^{2}$ & $\rho^{0(\mathrm{f})}=\hat{\rho}^{0(\mathrm{f})} v^{0(\mathrm{f})}=39 \mathrm{~kg} / \mathrm{m}^{3}$ & \\
& $R_{1}=2 \mathrm{~m}, R_{2}=20 \mathrm{~m}$ & \\
\hline
\end{tabular}

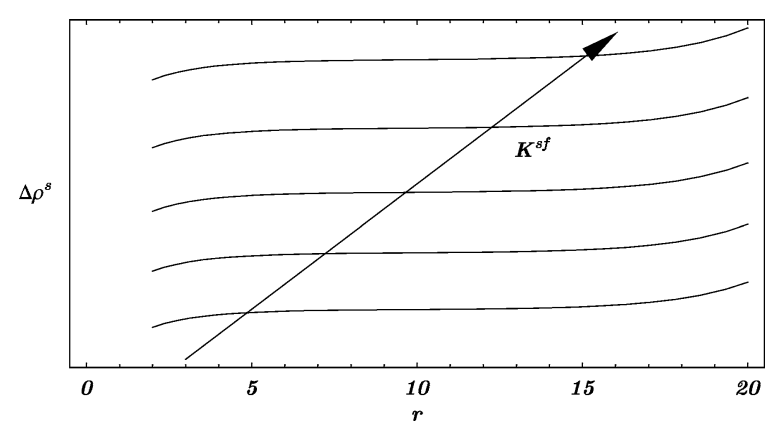

Figure 1. Qualitative $\Delta \rho^{(\mathrm{s})}$-profiles for $K^{\mathrm{sf}} \in\left(K_{1}^{\mathrm{sf}}, K_{2}^{\mathrm{sf}}\right)$.

applied double forces. Close to the boundary of the solid matrix their effect is either a dilatation or a compaction induced by the applied fluid pressure. In Figure 2 the solution apparently shows wide oscillations due to the change of type occurring in the Bessel equation (52). This is usually an indication of instability and motivates the following analysis.

\subsubsection{Stability of the Stressed Reference Configuration}

We now investigate the stability of the reference configuration with respect to changes in the coupling coefficient $K^{\text {sf }}$. Recalling our earlier remarks on the admissible values of $K^{\text {sf }}$ we delineate now the range of its values which assure the uniqueness of the solution of the elastic problem. Said differently, our goal is to characterize the coupling coefficient $K^{\mathrm{sf}}$ which ensures the structural stability of the partial differential equations defined on the space of state parameters $\mathbf{u}^{(\mathrm{s})}$ and $\rho^{(\mathrm{f})}$ and describing deformations of the mixture. One could also investigate the stability of the prestressed configuration with respect to the value of the prestress. According to the criterion stated, for example, by Arnold [2] or Thom [39], we dis- 


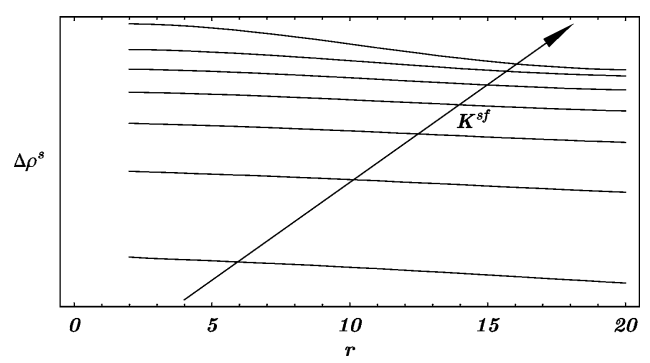

(a)

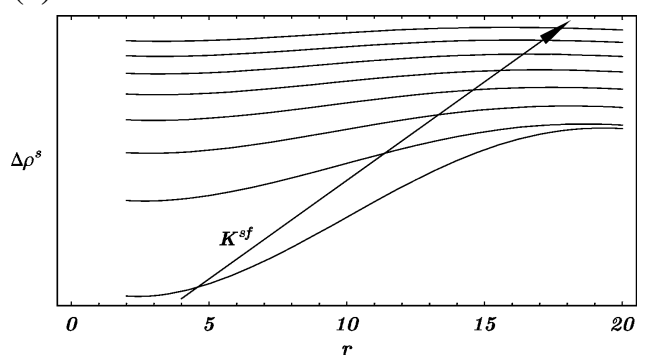

(b)

Figure 2. Qualitative $\Delta \rho^{(\mathrm{s})}$-profiles for $K^{\text {sf }} \in\left(-\infty, K_{1}^{\text {sf }}\right) \cup\left(K_{2}^{\text {sf }}, \infty\right)$. Figure 2(a) corresponds to $1.75<K<1.81$, and (b) to $1.83<K<1.9$, where $K=K^{\mathrm{sf}} /$ $\sqrt{\mu\left(2 \gamma^{0 \mathrm{f}} / \rho^{0}+\gamma^{\mathrm{ff}}\right)}$.

cuss the possibility that for two solutions corresponding to sufficiently close values of $K^{\text {sf }}$, an homeomorphism on the space of mixture states exists transforming one solution into the other. The norm induced by the energy inner product is used to define the neighborhood of an element in this space.

In order that the partial differential equations of our problem fulfill the condition of topological equivalence (see [2]) it is assumed that a solution of equations (47) and (48) describes available transformations of a reference configuration. This provides an admissible criterion for the stability analysis; many other choices are possible.

The following physically meaningful energetic criterion can be used to study the stability. It requires that for the reference equilibrium configuration to be stable, the total energy given by the functional

$$
\begin{aligned}
& \mathcal{E}_{\mathrm{tot}}\left(\rho^{(\mathrm{f})}, \mathbf{F}^{(\mathrm{s})}\right) \\
& \quad=\int_{\Omega}\left[\rho \epsilon\left(\rho^{(\mathrm{f})}, \mathbf{F}^{(\mathrm{s})}\right)+\frac{\lambda_{\mathrm{s}}}{2}\left|\nabla \rho^{(\mathrm{s})}\right|^{2}-\psi^{\mathrm{ext}}\left(\mathbf{x}, \rho^{(\mathrm{s})}, \rho^{(\mathrm{f})}, \nabla \rho^{(\mathrm{s})}\right)\right] \mathrm{d} V
\end{aligned}
$$

be minimum in the reference configuration. Here essential boundary conditions are assumed to be prescribed on the entire boundary. In particular, we prove that the reference configuration is stable when the coupling coefficient lies in a suitable subset of the open interval $\left(K_{1}^{\text {sf }}, K_{2}^{\text {sf }}\right)$. The mathematical reasoning parallels that 
given, for example, in [12]. That is, for the reference configuration to be stable, the second functional derivative of $\varepsilon_{\text {tot }}$ evaluated in the reference configuration must be positive definite. We shall prove that, when $\epsilon$ is prescribed by equation (26), this is equivalent to requiring that the following spectral problem has positive eigenvalues only.

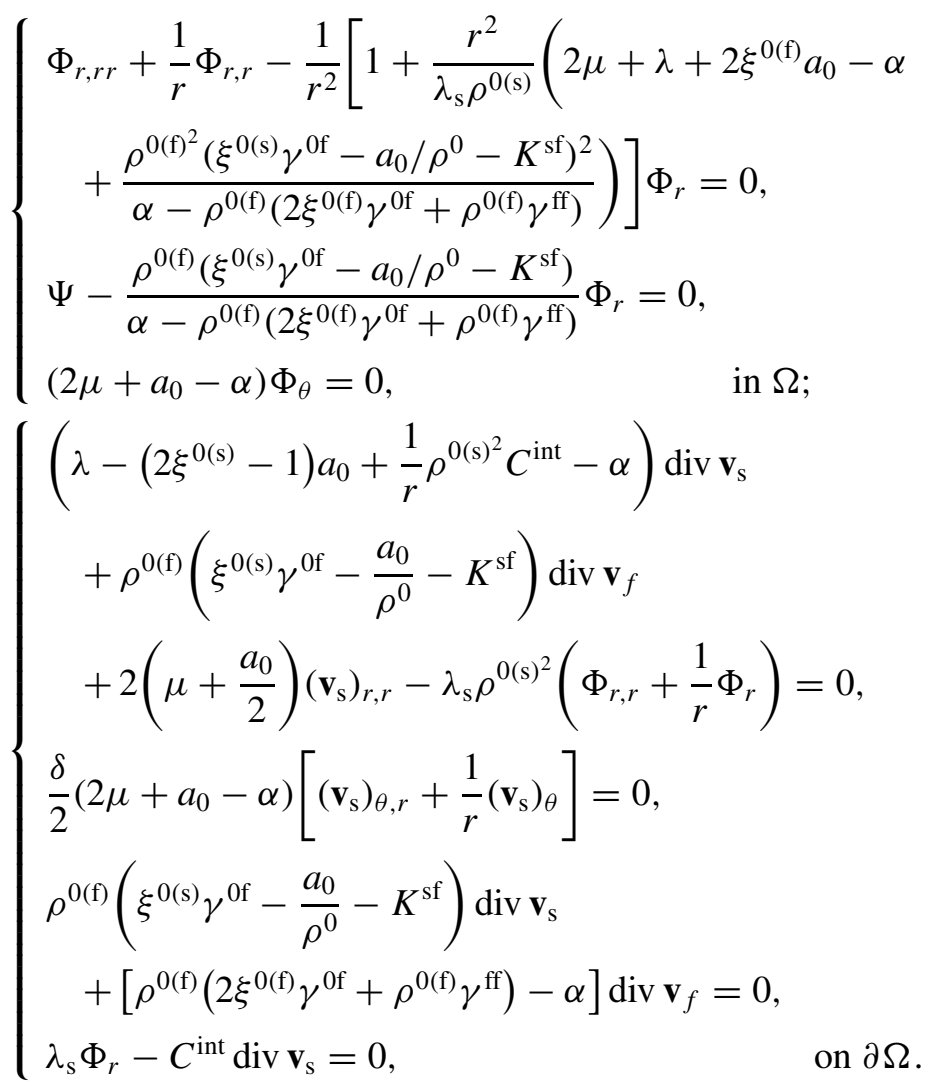

Here $\alpha$ denotes a generic eigenvalue, and quantities $\Phi_{r}, \Phi_{\theta}$ and $\Psi$ are defined by

$$
\begin{aligned}
\Phi_{r} & :=\nabla\left(\operatorname{div} \mathbf{v}_{\mathrm{s}}\right) \cdot \mathbf{e}_{r}=\left(\left(\mathbf{v}_{\mathrm{s}}\right)_{r, r}+\frac{1}{r}\left(\mathbf{v}_{\mathrm{s}}\right)_{r}\right)_{, r}, \\
\Phi_{\theta} & :=\operatorname{div}\left(\operatorname{skw}\left(\nabla \mathbf{v}_{\mathrm{s}}\right)\right) \cdot \mathbf{e}_{\theta}=\frac{1}{2}\left(\left(\mathbf{v}_{\mathrm{s}}\right)_{\theta, r}+\frac{1}{r}\left(\mathbf{v}_{\mathrm{s}}\right)_{\theta}\right)_{, r}, \\
\Psi & :=\nabla\left(\operatorname{div} \mathbf{v}_{f}\right) \cdot \mathbf{e}_{r},
\end{aligned}
$$

in terms of the virtual velocity fields $\mathbf{v}_{\mathrm{s}}$ and $\mathbf{v}_{f}$. This equivalence can be proved by following the procedure adopted by Seppecher [36]. In a virtual motion the second time derivative of $\varepsilon_{\text {tot }}$ evaluated in the reference configuration is required to equal the integral over $\Omega$ of a suitable quadratic form multiplied by $\alpha$. Consequently its sign depends on the sign of $\alpha$. In order to get conditions for a positive definite 
second derivative of $\varepsilon_{\text {tot }}$ by means of a suitable eigenvalue problem, the proper quadratic form is

$$
\left.\frac{\mathrm{d}^{2} \varepsilon_{\text {tot }}}{\mathrm{d} t^{2}}\right|_{\left(\rho^{0(\mathrm{f})}, \rho^{0(\mathrm{~s})}, \bar{p}_{0}\right)}=\int_{\Omega} \alpha\left[\left(\operatorname{div} \mathbf{v}_{\mathrm{s}}\right)^{2}+\operatorname{skw}\left(\nabla \mathbf{v}_{\mathrm{s}}\right) \cdot \operatorname{skw}\left(\nabla \mathbf{v}_{\mathrm{s}}\right)+\left(\operatorname{div} \mathbf{v}_{f}\right)^{2}\right] \mathrm{d} V
$$

It is clear that if $\alpha$ is positive then the left-hand side of equation (58) is positive and the reference configuration is stable. Cumbersome calculations involved in the derivation of equations (55) and (56) from equation (58) have been omitted. We simply note that the divergence theorem applied to the right-hand side of equation (58) implies that field equations (55) and boundary conditions (56) depend on the eigenvalue $\alpha$.

Field equation (55) 1 is a Bessel equation. Therefore, according to the sign of

$$
Q:=\frac{1}{\lambda_{\mathrm{s}} \rho^{0(\mathrm{~s})^{2}}}\left(2 \mu+\lambda+2 \xi^{0(\mathrm{f})} a_{0}-\alpha+\frac{\rho^{0(\mathrm{f})^{2}}\left(\xi^{0(\mathrm{~s})} \gamma^{0 \mathrm{f}}-a_{0} / \rho^{0}-K^{\mathrm{sf}}\right)^{2}}{\alpha-\rho^{0(\mathrm{f})}\left(2 \xi^{0(\mathrm{f})} \gamma^{0 \mathrm{f}}+\rho^{0(\mathrm{f})} \gamma^{\mathrm{ff}}\right)}\right)
$$

its solution is a linear combination of classical Bessel functions or of modified Bessel functions. In particular, if $Q<0$ the solution of equation (55) 1 is a linear combination of $J_{1}(\sqrt{-Q} r)$ and $Y_{1}(\sqrt{-Q} r)$, conversely if $Q>0$ it is a linear combination of $I_{1}(\sqrt{Q} r)$ and $K_{1}(\sqrt{Q} r)$. In order to determine the range of $K^{\text {sf }}$ for which eigenvalues are positive, we first determine the range of eigenvalues corresponding to positive or negative values of $Q$. When $\alpha$ is negative, we restrict our discussion to the case

$$
\alpha<\underbrace{\rho^{0(\mathrm{f})}\left(2 \xi^{0(\mathrm{f})} \gamma^{0 \mathrm{f}}+\rho^{0(\mathrm{f})} \gamma^{\mathrm{ff}}\right)}_{>0} .
$$

Consequently we get

$$
\begin{aligned}
& Q>0 \quad \Rightarrow \quad \alpha<\alpha_{1}, \alpha>\alpha_{2}, \\
& Q<0 \quad \Rightarrow \quad \alpha_{1}<\alpha<\alpha_{2},
\end{aligned}
$$

where $\alpha_{1}$ and $\alpha_{2}$ are roots of the equation $Q=0$. Consider the case when $\alpha<\alpha_{1}$; it is easy to check that, for values of the constitutive coefficients considered in Table I, a characteristic root always exists in this range of eigenvalues. Therefore solutions of equations (55) are linear combinations of modified Bessel functions. 


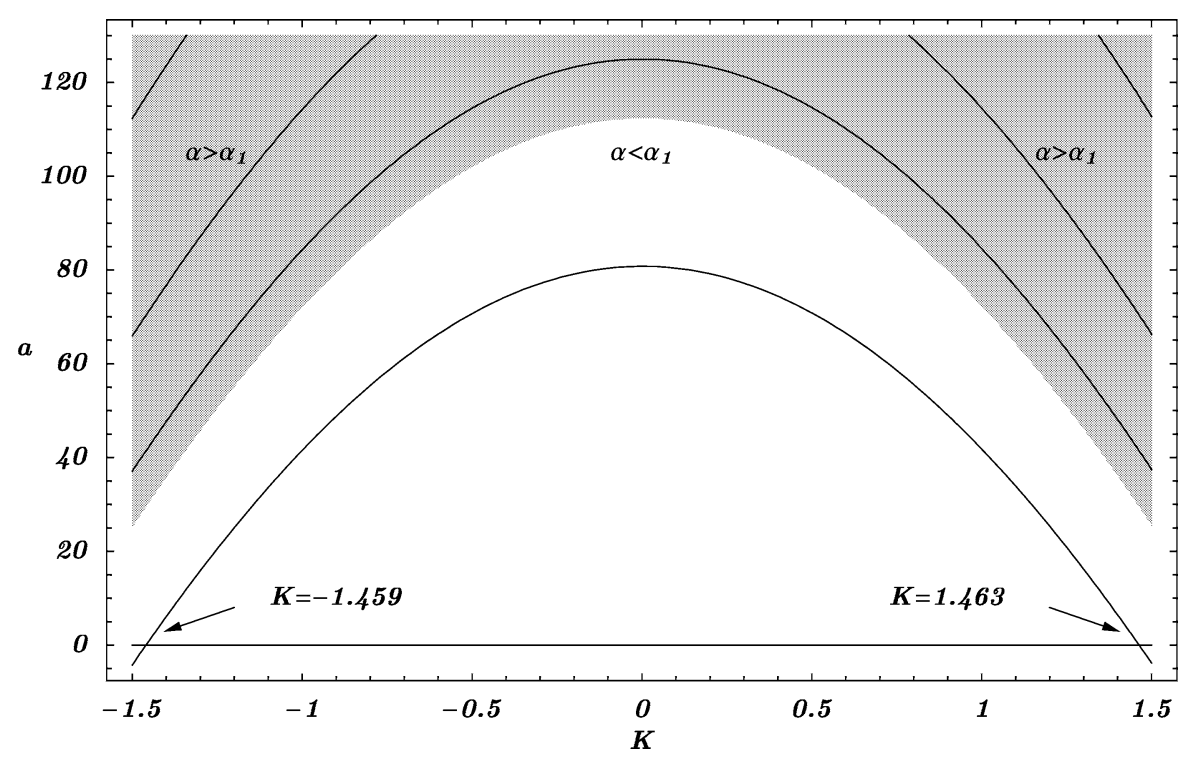

Figure 3. The solution of equation $\varphi\left(\alpha, K^{\mathrm{sf}}\right)=0$ for $\alpha<\alpha_{1}$ is presented where $a=$ $\alpha / \lambda_{\mathrm{s}} \rho^{0(\mathrm{~s})^{2}}$.

Assume that $\alpha \neq 2 \mu+a_{0}$, i.e., require equation $(55)_{3}$ to be satisfied by a rigid body rotation. Then equations (55) imply that

$$
\begin{aligned}
\Phi_{r} & =C_{1} I_{1}(\sqrt{Q} r)+C_{2} K_{1}(\sqrt{Q} r), \\
\Phi_{\theta} & =0, \\
\operatorname{div} \mathbf{v}_{\mathrm{s}} & =\frac{C_{1}}{\sqrt{Q}} I_{0}(\sqrt{Q} r)-\frac{C_{2}}{\sqrt{Q}} K_{0}(\sqrt{Q} r)+C_{3}, \\
\left(\mathbf{v}_{\mathrm{s}}\right)_{r} & =\frac{C_{1}}{Q} I_{1}(\sqrt{Q} r)+\frac{C_{2}}{Q} K_{1}(\sqrt{Q} r)+\frac{C_{3}}{2} r+\frac{C_{4}}{r},
\end{aligned}
$$

where $C_{i}(i=1, \ldots, 4)$ are constants of integration. Substituting from (62) into the homogeneous boundary conditions (56) and requiring that the determinant of this linear system vanish, one gets the equation which determines the admissible eigenvalues of the system for $\alpha<\alpha_{1}$. These solutions are functions of the coupling coefficient $K^{\text {sf }}$. Let $\varphi$ be the determinant of the linear system obtained from equations (56) and regarded as a function of $\alpha$ and $K^{\text {sf }}$. In Figure 3 the curve $\varphi\left(\alpha, K^{\text {sf }}\right)=0$ is exhibited. The gray area in the figure indicates the region of positive eigenvalues larger than $\alpha_{1}$. For values of the coupling coefficient in the range $\left(K_{1}^{\text {sf }}, K_{2}^{\text {sf }}\right)$ an eigenvalue smaller than $\alpha_{1}$ always exists. Consequently, the loss of stability of the equilibrium configuration occurs when such an eigenvalue becomes negative. A numerical simulation shows that the stability is guaranteed for values of the coupling coefficient in a suitable open subset $\left(K_{1 \mathrm{~s}}^{\mathrm{sf}}, K_{2 \mathrm{~s}}^{\mathrm{sf}}\right)$ of $\left(K_{1}^{\mathrm{sf}}, K_{2}^{\mathrm{sf}}\right)$. Thus solutions of equations (47) and (48) are meaningful only for coupling coefficients in this open interval. 

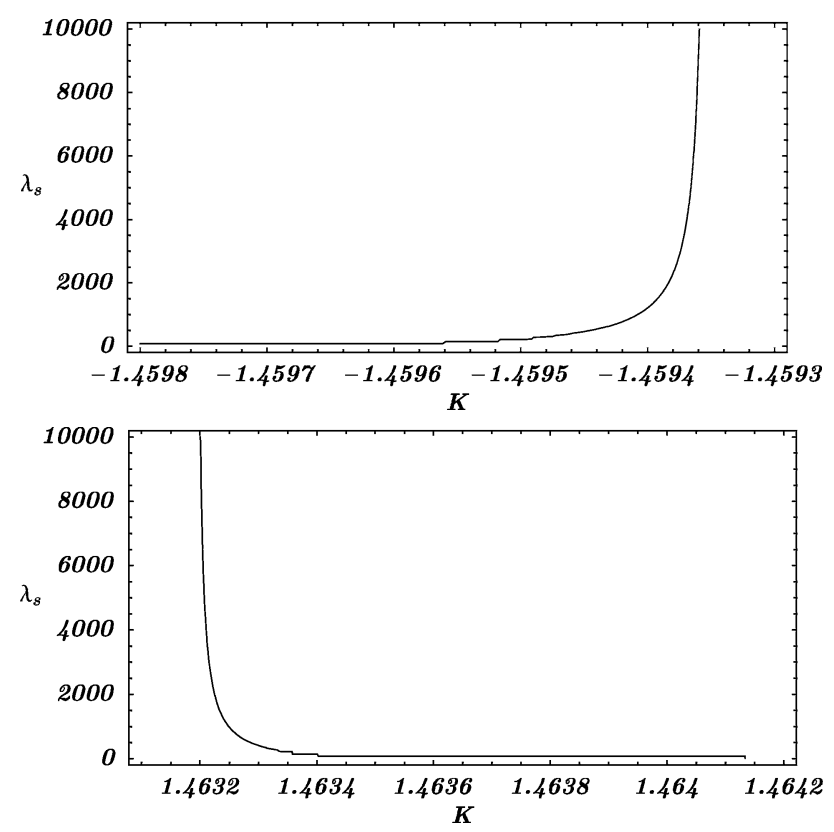

Figure 4. Dependence of $K_{1 \mathrm{~s}}^{\mathrm{sf}}$ and $K_{2 \mathrm{~s}}^{\mathrm{sf}}$ upon $\lambda_{\mathrm{s}}$.

These results indicate that the stability-instability transition does not involve a change in the macroscopic deformation profile for the hollow cylinder. In other words the loss of stability and wide oscillations occurring when the coupling coefficient belongs to the open set $\left(-\infty, K_{1}^{\text {sf }}\right) \cup\left(K_{2}^{\text {sf }}, \infty\right)$ are not correlated. This could be due to the stability criterion used here. A nonlinear analysis and/or a different choice of the energetic functional may provide a different stability limit.

It is interesting to note that the stability limits $K_{1 \mathrm{~s}}^{\mathrm{sf}}$ and $K_{2 \mathrm{~s}}^{\mathrm{sf}}$ are not strongly affected by second gradient effects. An increase in $\lambda_{\mathrm{s}}$ does not induce a noticeable change in these limits (see Figure 4). However, estimates of the stability limits provided by the first gradient theory (i.e. $\lambda_{\mathrm{s}}=0$ ) cannot be used when second gradient effects are present, and the length of the stable region progressively decreases as second gradient effects become more relevant (see Figure 4).

\section{Concluding Remarks}

We have studied infinitesimal static deformations of a long hollow porous isotropic elastic cylinder initially saturated with a perfect fluid and subjected to a hydrostatic pressure on the inner and the outer surfaces. The cylinder in the reference configuration is stressed. Equations governing deformations of the solid and the fluid that are linear in displacement gradients and infinitesimal changes in the apparent mass densities of the solid and the fluid have been derived; these are Heun's equations and may have singular points in the interior of the hollow cylinder. Constitutive 
relations for the solid have been assumed to depend upon the gradients of the apparent mass density of the solid. Deformations of the solid and the fluid are coupled through the scalar coefficient $K^{\text {sf }}$ multiplying gradients of the apparent mass density of the solid. When the initial stress state in the solid is that of uniform pressure, equations governing the radial and the circumferential components of displacement are uncoupled; the former is a nonhomogeneous Bessel equation and the latter an Euler equation. These equations are solved for a somewhat arbitrarily chosen set of material and geometric parameters; these correspond to a salt matrix filled with brine. The stability of the reference configuration with respect to changes in the values of the coupling coefficient $K^{\text {sf }}$ has been scrutinized. It is found that the reference configuration is stable for values of the coupling coefficient in a suitable open set. The computed profiles of the mass density of the solid phase exhibit an oscillatory behavior and also a boundary layer near the outer surface. The variation of changes in the apparent mass density of the solid due to the consideration of second-gradient effects is about $1 \%$ of its value in the absence of second-gradient effects and depends upon the value assigned to the coupling coefficient $K^{\text {sf }}$.

\section{References}

1. G.E. Andrews, R. Askey and R. Roy, Special functions. In: Encyclopedia of Mathematics and Its Applications. Cambridge Univ. Press., Cambridge (1999).

2. V.I. Arnold, Geometrical Methods in the Theory of Ordinary Differential Equations, translated by J. Szucs; English translation edited by M. Levi. Springer, New York (1983).

3. F.M. Arscott, Heun's equation. In: A. Ronveaux (ed.), Heun's Differential Equations. Oxford Univ. Press, Oxford (1995) Part A.

4. R.C. Batra, On nonclassical boundary conditions. Arch. Rational Mech. Anal. 48 (1972) 163191.

5. R.C. Batra, Thermodynamics of non-simple elastic materials. J. Elasticity 6 (1976) 451-456.

6. R.C. Batra, The initiation and growth of, and the interaction among adiabatic shear bands in simple and dipolar materials. Internat. J. Plasticity 3 (1987) 75-89.

7. R.C. Batra and L. Chen, Shear band spacing in gradient-dependent thermoviscoplastic materials, Comput. Mech. 23 (1999) 8-19.

8. R.C. Batra and J. Hwang, Dynamic shear band development in dipolar thermoviscoplastic materials. Comput. Mech. 12 (1994) 354-369.

9. R.C. Batra and C.H. Kim, Adiabatic shear banding in elastic-viscoplastic nonpolar and dipolar materials. Internat. J. Plasticity 6 (1990) 127-141.

10. P. Bérest, J. Bergues, B. Brouard, J.G. Durup and B. Guerber, A salt cavern abandonment test. Internat. J. Rock Mech. Min. 38 (2001) 357-368.

11. M.A. Biot, General theory of three-dimensional consolidation. J. Appl. Phys. 12 (1941) 155164.

12. P. Blanchard and E. Bruning, Variational Methods in Mathematical Physics (a Unified Approach). Springer, Heidelberg (1992).

13. R.M. Bowen, Theory of mixtures. In: Continuum Physics, Vol. III (1976) pp. 2-127.

14. J.W. Cahn and J.E. Hilliard, Free energy of a non-uniform system. J. Chem. Phys. 31 (1959) 688-699.

15. P. Casal, La théorie du second gradient et la capillarité. C. R. Acad. Sci. Paris Sér. A 274 (1972) 1571-1574. 
16. P. Casal and H. Gouin, Equations du movement des fluides thermocapillaires. C. R. Acad. Sci. Paris Sér. II 306 (1988) 99-104.

17. P. Cosenza, M. Ghoreychi, B. Bazargan-Sabet and G. de Marsily, In situ rock salt permeability measurement for long term safety assessment of storage. Internat. J. Rock Mech. Min. 36 (1999) 509-526.

18. R. de Boer, Theory of Porous Media. Springer, Berlin (2000).

19. F. dell'Isola, M. Guarascio and K. Hutter, A variational approach for the deformation of a saturated porous solid. A second gradient theory extending Terzaghi's effective stress principle. Archive Appl. Mech. 70 (2000) 323-337.

20. F. dell'Isola and P. Seppecher, Edge contact forces and quasibalanced power. Meccanica 32 (1997) 33-52.

21. W. Elhers, Toward finite theories of liquid-saturated elasto-plastic porous media. Internat. J. Plasticity 7 (1991) 433-475.

22. P. Fillunger, Erdbaumechanik. Selbstverlag des Verfassers, Wien (1936).

23. P. Germain, La méthode des puissances virtuelles en mécanique des milieux continus. J. Mécanique 12(2) (1973) 235-274.

24. P. Germain, The method of virtual power in continuum mechanics. Part 2: Microstructure. SIAM J. Appl. Mech. 25(3) (1973) 556-575.

25. M.A. Goodman and S.C. Cowin, A continuum theory for granular materials. Arch. Rational Mech. Anal. 44 (1972) 249-266.

26. H. Gouin, Tension superficielle dynamique et effet Marangoni pour les interfaces liquidevapeur en théorie de la capillarité interne. C. R. Acad. Sci. Paris Sér. II 303(1) (1986).

27. S. Krishnaswamy and R.C. Batra, A thermomechanical theory of solid-fluid mixtures. Math. Mech. Solids 2 (1997) 143-151.

28. G.A. Maugin, The method of virtual power in continuum mechanics: application to coupled fields. Acta Mech. 35 (1980) 1-70.

29. L.W. Morland, A simple constitutive theory for a fluid saturated porous solid. J. Geoph. Res. 77 (1972) 890-900.

30. I. Müller, A thermodynamic theory of mixtures of fluids. Arch. Rational Mech. Anal. 28 (1968) $1-39$.

31. I. Müller, Thermodynamics. Pittman, Boston (1985).

32. N.I. Muskhelishvili, Some Basic Problems of the Mathematical Theory of Elasticity. Noordorf, Groningen (1953).

33. K.R. Rajagopal and L. Tao, Mechanics of Mixtures. World Scientific, Singapore (1995).

34. G. Sciarra, F. dell'Isola and K. Hutter, A solid-fluid mixture model allowing for solid dilatation under external pressure. Continuum Mech. Thermodyn. 13 (2001) 287-306.

35. G. Sciarra, K. Hutter and G.A. Maugin, A variational approach to a micro-structured theory of solid-fluid mixtures, in preparation.

36. P. Seppecher, Equilibrium of a Cahn-Hilliard fluid on a wall: Influence of the wetting properties of the fluid upon the stability of a thin liquid film. European J. Mech. B Fluids 12(1) (1993) 69-84.

37. S.M.R.I. Solution, Mining Research Institute, Technical class guidelines for safety assessment of salt caverns, Fall Meeting, Rome, Italy (1998).

38. B. Svendsen and K. Hutter, On the thermodynamics of a mixture of isotropic materials with constraints. Internat. J. Engrg. Sci. 33 (1995) 2021-2054.

39. R. Thom, Stabilité structurelle et morphogénèse: Essai d'une Théorie Générale des Modèles. Benjamin, New York (1972).

40. C.A. Truesdell, Sulle basi della termomeccanica. Lincei Rend. Sc. Fis. Mat. Nat. XXII (Gennaio 1957).

41. C.A. Truesdell, Thermodynamics of diffusion, Lecture 5. In: C.A. Truesdell (ed.), Rational Thermodynamics. Springer, Berlin (1984) pp. 216-219. 